\title{
Orbit-Reversing Magnets for the National Bureau of Standards-Los Alamos Racetrack Microtron
}

\author{
M. A. WILSON, P. H. DEBENHAM, S. PENNER, AND S. S. BRUCE
}

\begin{abstract}
In the National Bureau of Standards (NBS)-Los Alamos racetrack microtron (RTM), the 17-MeV electron beam that has made one pass through the RTM linac is deflected $180^{\circ}$ in one end magnet and is returned to the same end of the (standing wave) linac for a second pass. $A$ pair of dipole magnets on the linac axis compensate for the beam displacement caused by the end magnet so that the beam enters the linac on axis. These two magnets are designed to have equal field integrals in order to produce a pure displacement. Matching the field integrals was complicated by the quite different widths of the two magnets that have different beam clearance requirements. In addition the wider magnet contains a quadrupole coil for beam steering. Design considerations are presented. Magnetic field measurements show that critical design goals have been achieved.
\end{abstract}

\section{Description AND Design ReQUIREMENTS}

$F$ IG. 1 shows the National Bureau of Standards (NBS)-Los Alamos racetrack microtron (RTM) [1]. The 5-MeV beam from the injector linac [2] is injected into the RTM linac at the right side of Fig. 1 and is accelerated to $17 \mathrm{MeV}$ in the first pass through the linac. Since the linac is a standing-wave radio frequency (RF) structure it can accelerate beams in either direction. The $17-\mathrm{MeV}$ beam is deflected through $180^{\circ}$ in the end magnet E1 for a second pass through the linac in the reverse direction. The function of dipole magnets D7 and D8 is to compensate for the displacement of the $17-\mathrm{MeV}$ orbit by E1 and return the beam to the linac axis, as shown in Fig. 2. The recirculating beam at energies of $29 \mathrm{MeV}$ and above bypasses the linac in the return lines and is returned to the linac axis for recirculation through the linac by magnets E1, D8, and D7, as shown in Fig. 2. In order to keep the contribution of magnet power supply current noise to beam emittance negligible, magnets D7 and D8 are connected in series to a common power supply having $10^{-3}$ regulation and are designed to produce equal integrated magnetic fields to within $\pm 30 \mathrm{G} \cdot \mathrm{cm}$. The net deflection of D7 and D8 is compensated with steerers on the return lines and the displacement with chicane magnets [3] D9 and D10 (see Fig. 1).

Because magnets D7 and D8 bend the 17-Mev beam in both forward and reverse directions, the net angular deflection at 17 $\mathrm{MeV}$ is zero if the magnetic fields are perfectly uniform. Some angular deflection of the $17-\mathrm{MeV}$ beam is required to compensate for misalignments and the local obliquity of the effective edge of end magnet E1 caused by the pole corner [4]. To provide the required deflection, an independently variable quadrupole field of $\pm 1 \mathrm{G} \cdot \mathrm{cm}^{-1}$ is included in D8.

Manuscript received June 13, 1988. This work supported in part by the U.S. Department of Energy.

The authors are with the Radiation Source and Instruments Division, National Bureau of Standards, Gaithersburg, MD 20899.

IEEE Log Number 8825401.
The aperture of D8 must accommodate extracted beam orbits as well as the $17-\mathrm{MeV}$ orbit. In contrast, the aperture of D7 must be narrow to avoid interference with magnet D12 in the extraction line. Finally, the relative sextupole coefficient of the fields of D7 and D8 must be less than $7 \times 10^{-4} \mathrm{~cm}^{-2}$ to limit emittance growth to an acceptable level.

\section{DESIGN RESULtS}

Due to space constraints a picture-frame design was selected. In the magnet design process, two-dimensional magnetic field calculations were preformed for both transverse and longitudinal cross sections using the magnet code TRIM [5]. Figs. 3 and 4 show schematic cross sections of the two magnets. Yokes of fully annealed very low carbon steel, 1010 or lower, are necessary to achieve the required field uniformly in the TRIM calculation. A quadrupole component has been designed into the field of D8 by including Panofsky uniform current sheets in addition to the dipole coils [6]. Due to the limited space between the yoke and the vacuum chamber, the current sheets must be quite thin with a high conductor packing factor. Due to the large gap width to height ratio in D8, one leg of the current sheet will be much narrower than the other, yet it must carry the same current. The cross section of the upper half of D8 including the dipole and quadrupole coils is shown in Fig. 4. A field plot calculated with TRIM for this coil arrangement is also shown. Fig. 5 is a picture of one of the four coils before bending. This sheet contains 10 turns of $140-\mu \mathrm{m}$ copper sandwiched between $51-\mu \mathrm{m}$ sheets of kapton.

In order to achieve equal field integrals in D7 and D8, the yoke of D8 was made thicker to reduce the reluctance due to the longer path length, and the gap in D7 was made $0.4 \mathrm{~mm}$ larger. The completed magnets are shown in Fig. 6.

\section{Field Measurements}

The field of the completed magnets has been measured using a small table-top field mapper with a Hall probe field monitor. The probe is manually positioned with precision screw drives. The probe position is determined in the horizontal $(x-z)$ plane by linear scales read optically to an accuracy of $0.01 \mathrm{~mm}$. Field and position data are recorded on a floppy disk directly from the instruments and then printed out for evaluation. Fig. 7 shows the measured field in D8 plotted for the transverse cross section without the quadrupole coils. The dipole results from the TRIM calculations are included for comparison. The measured and design field values agree to 0.6 percent, and the measured sextupole term 


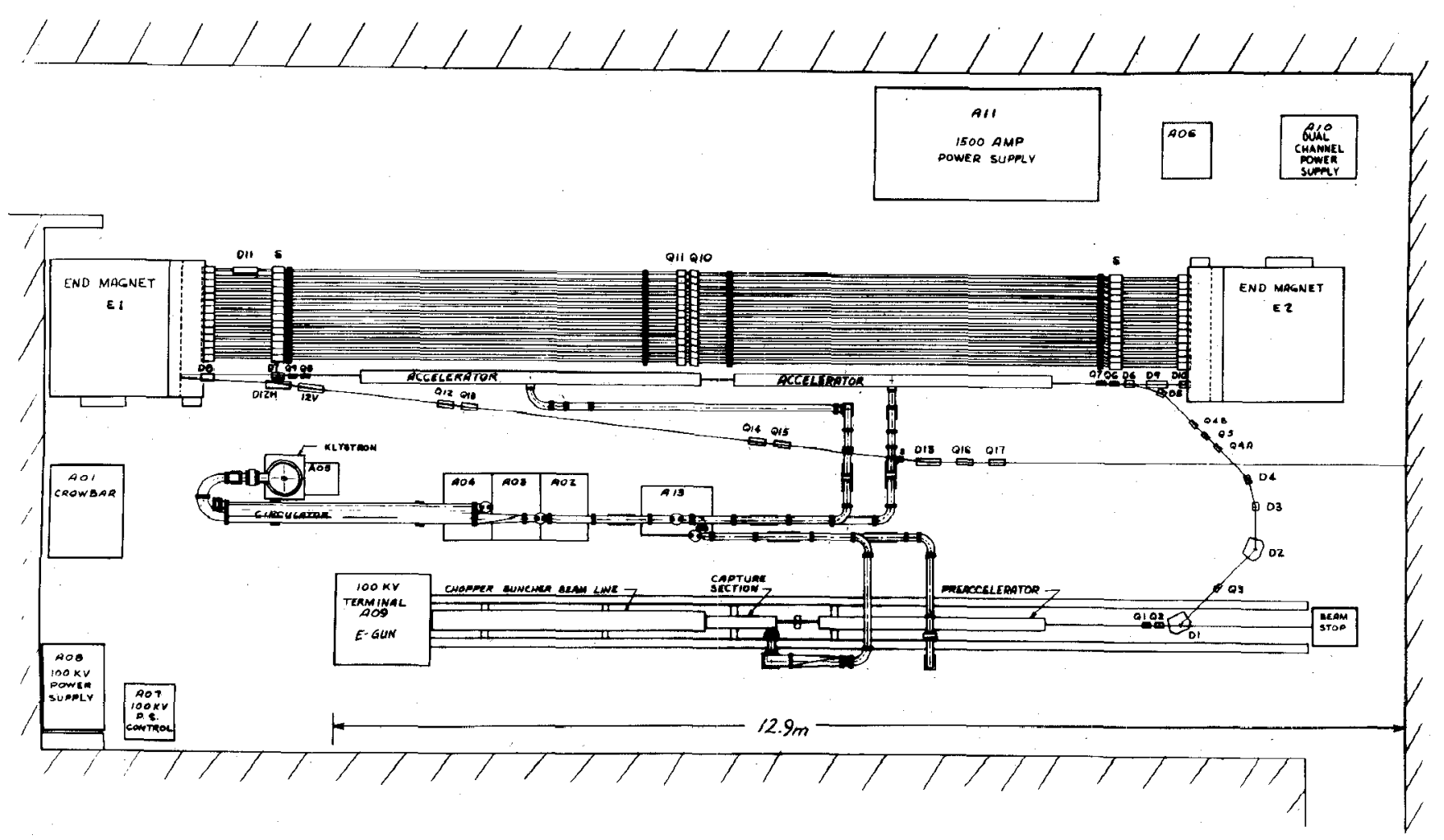

Fig. 1. Plan view of NBS-Los Alamos racetrack microtron (RTM).

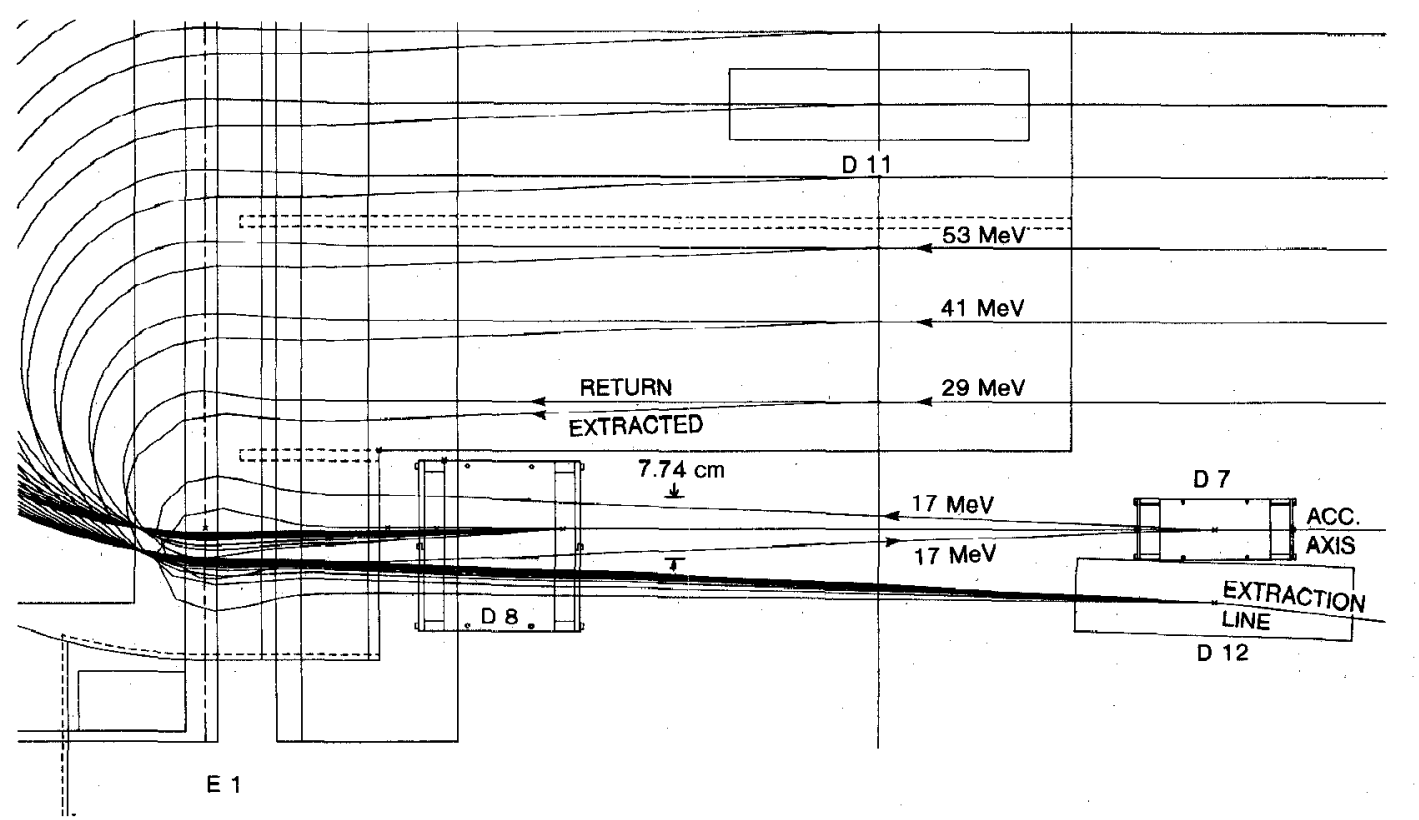

Fig. 2. Orbit-reversing region of RTM. Orbits for both recirculation and extraction are shown. Magnet D11 can be moved to any return line for extraction. 


\section{D7 TRANSVERSE CROSS SECTION}

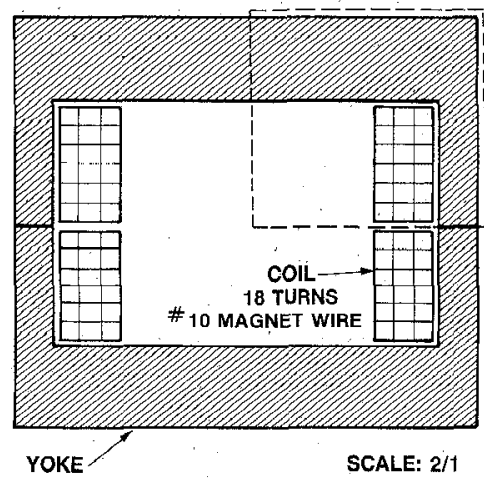

Fig. 3. Cross section of D7.

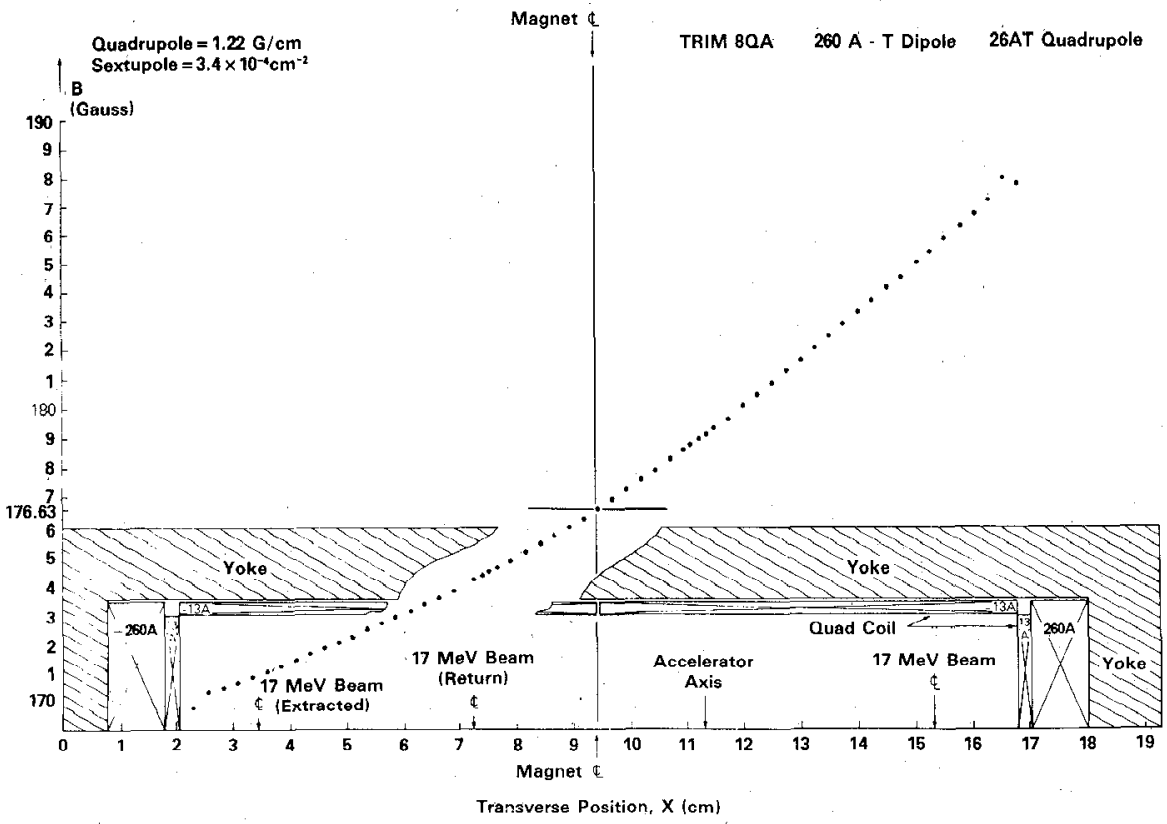

Fig. 4. Cross section of upper half of D8 and field plot from TRIM including quadrupole.

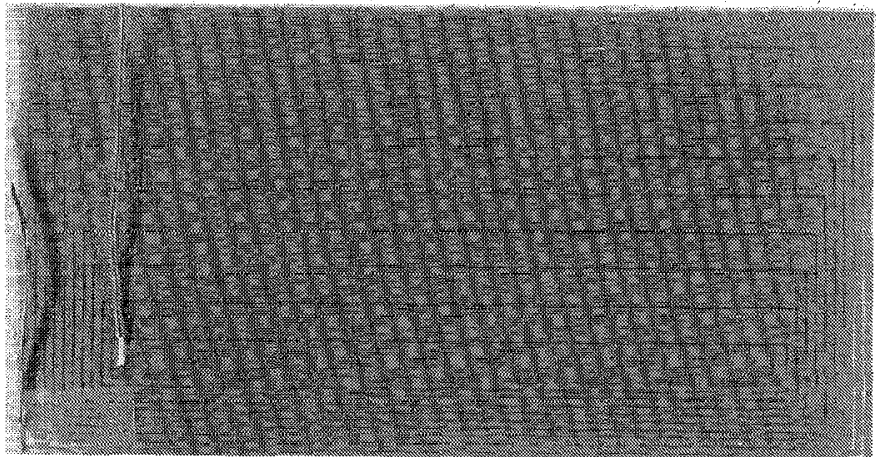

Fig. 5. 10-turn quadrupole sheet coil before bending.

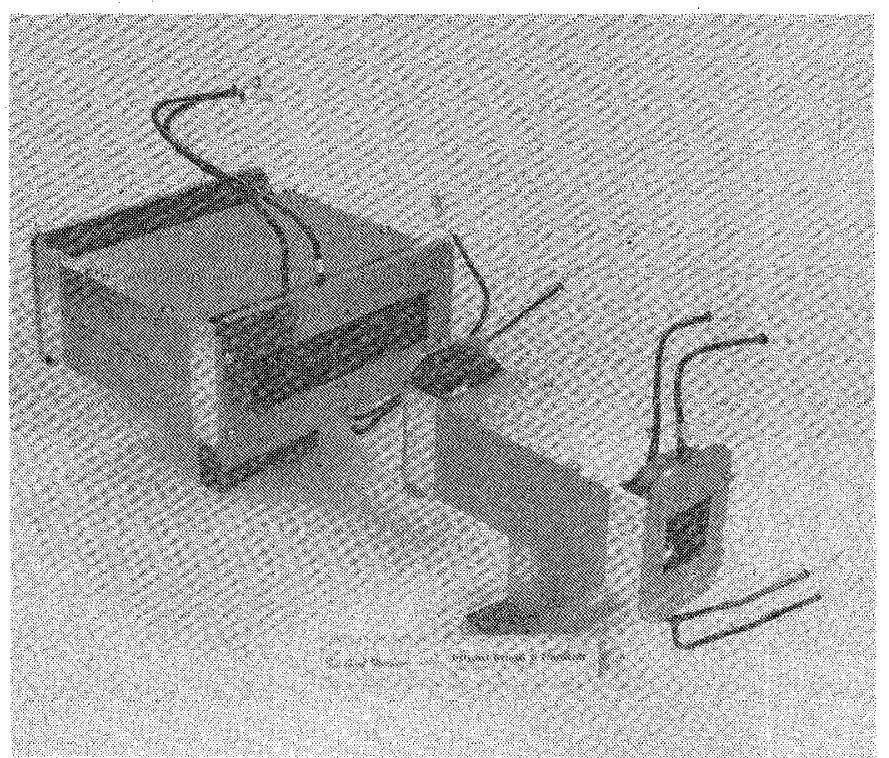

Fig. 6. Photo of D7 (left) and D8 (right). 


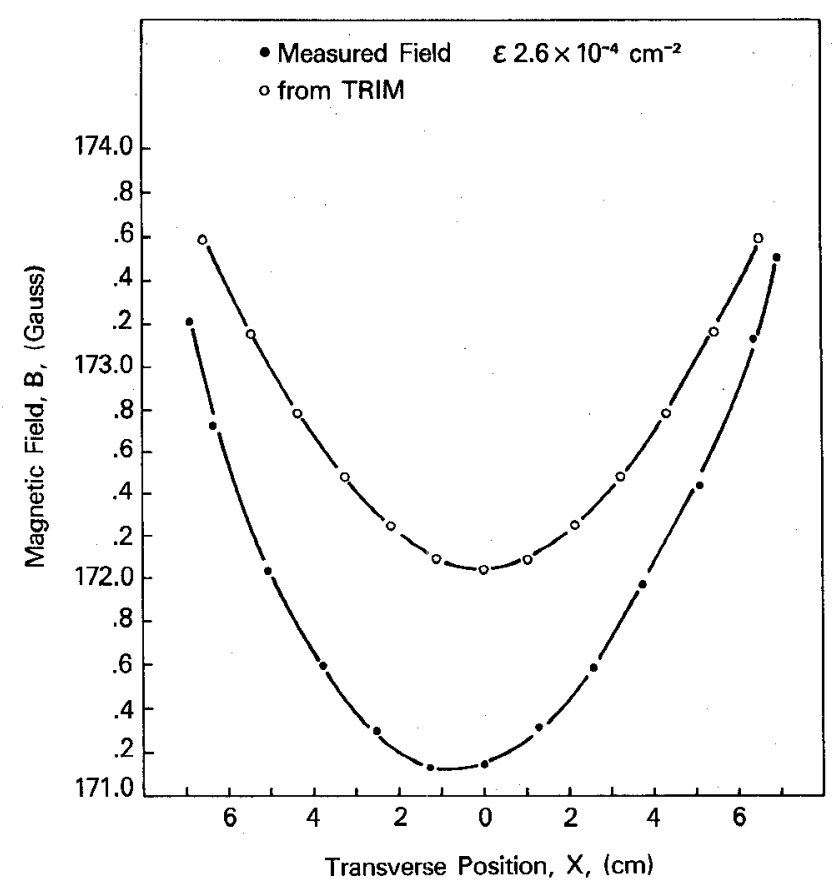

Fig. 7. Measured field near the middle of $D 8$ versus transverse position, compared to TRIM results (open circles).

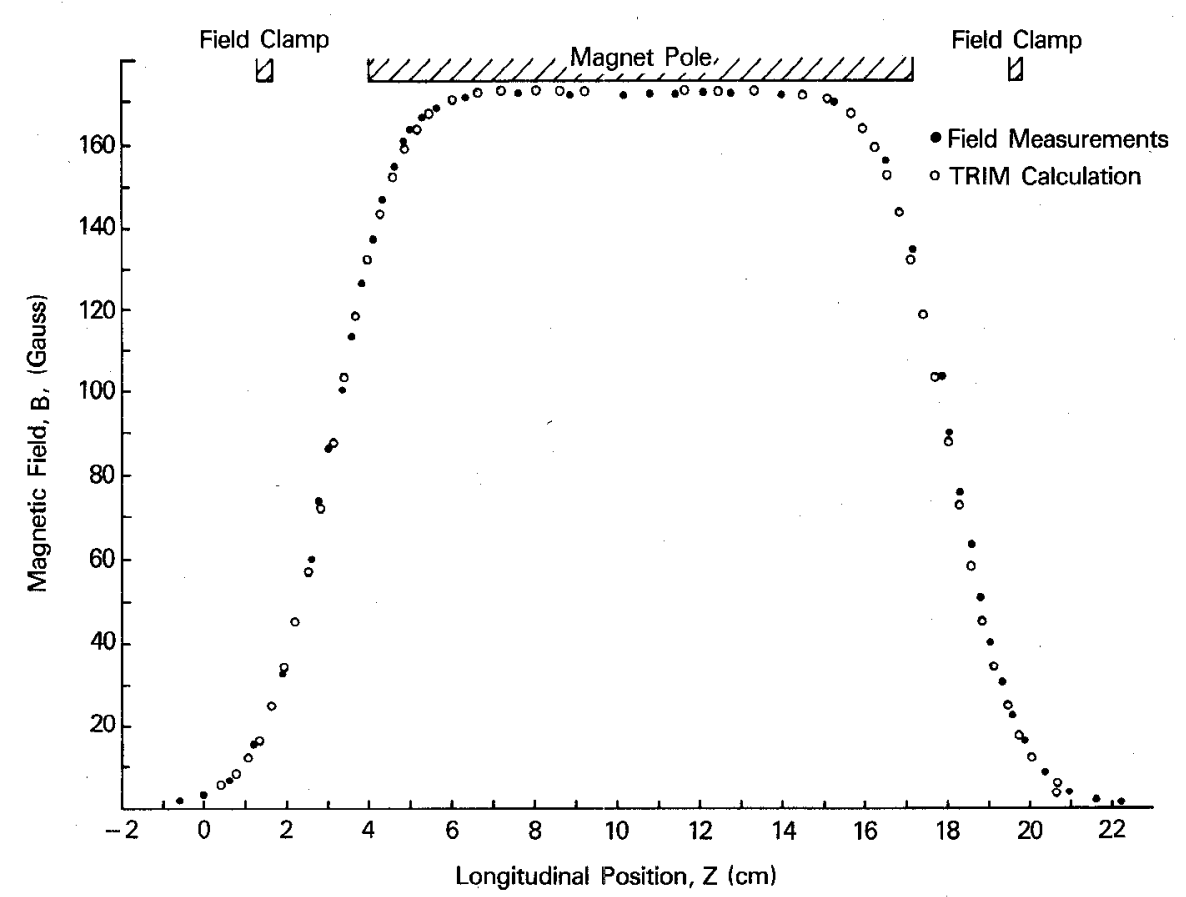

Fig. 8. Measured field in D8 plotted along the longitudinal axis. Open circles are TRIM results.

is well within the allowed limit. Recent field measurements with the quadrupole coils energized show a transverse gradient in the field which is within 3 percent of the gradient calculated with TRIM, as shown in Fig. 4. The measured and calculated fields plotted with respect to the longitudinal magnet axis of D8 are shown in Fig. 8. The measured field integral along the $z$ axis is $2611 \mathrm{G} \cdot \mathrm{cm}$. From similar measurements of D7, the field integral is only $4 \mathrm{G} \cdot \mathrm{cm}$ less than for D8. This difference is well within the design criterion of $30 \mathrm{G} \cdot \mathrm{cm}$.

Measured field plots of the transverse cross section of D7 are shown in Fig. 9 in which the sensitivity of the field uniformity to coil position is also demonstrated. By adjusting the coil positions by only $0.3 \mathrm{~mm}$ (corresponding to 2 percent of the magnet field width) symmetrically about the midplane, the sextupole term can be reversed in sign and reduced by an order of magnitude. The results of TRIM calculations for coils positioned in the middle of each half-gap are shown for comparison in Fig. 9(b) with the coil position producing the flattest field.

The measured current stability of the power supply for D7 


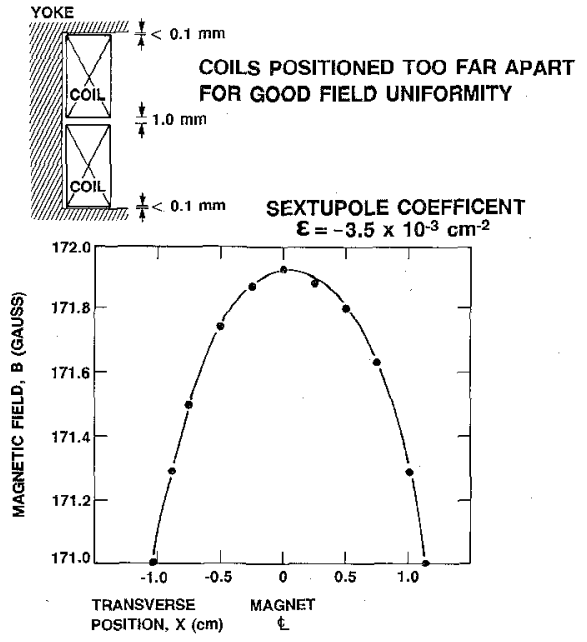

(a)

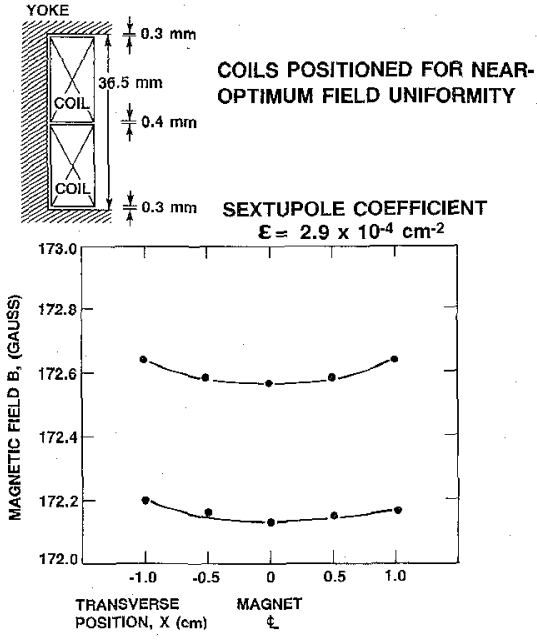

(b)

Fig. 9. Measured field in D7 plotted along the transverse axis for two coil positions. Upper curve in 9(b) is the TRIM result for the coils centered in the upper and lower half-gaps.

and D8 is \pm 0.01 percent (60-Hz ripple) which is well within the design requirement.

\section{Summary AND CONCLUSION}

A pair of dipole magnets that return the reversed orbit of the 17-MeV electron beam to the RTM linac axis for another pass through the linac have been designed, constructed, and measured. Field integral differences between the two magnets and field uniformity of both magnets are well within design limits. Field uniformity was demonstrated to be quite sensitive to coil position in narrow window magnets. A simple flexible thin high-current coil in the configuration of a Panofsky uniform current sheet has been developed for insertion into a dipole window magnet to produce an independently variable quadrupole field.

\section{ACKNOWLEDGMENT}

The assistance of Eric Lindstrom in implementing the program for calculating field integrals is gratefully acknowledged.

\section{REFERENCES}

[1] S. Penner et al., "Progress report on the NBS-Los Alamos racetrack microtron," IEEE Trans. Nucl. Sci., vol. NS-32, no. 5, p. 2669, Oct. 1985.

[2] M. A. Wilson et al., "Performance of the 5-MeV injector for the NBSLos Alamos racetrack microtron," in Proc. 1987 IEEE Particle Accelerator Conference, p. 322.

[3] P. H. Debenham et al., "Injection chicane magnets for a racetrack microtron," in Proc. 1987 IEEE Particle Accelerator Conference, p. 1452 .

[4] P. H. Debenham et al., "End magnets for the NBS-Los Alamos racetrack microtron," IEEE Trans. Nucl. Sci., vol. NS-32, no. 5, p. 3648 , Oct. 1985.

[5] A. M. Winslow, J. Comput. Phys., vol. 1, p. 149, 1966.

[6] L. N. Hand and W. K. Panofsky, Rev. Sci Inst., vol. 30, p. 927, 1959. 